Equipo Arquitectura

\title{
PREVI Lima: 35 años después
}

Adaptarse a las necesidades del habitante, y dar espacio a sus aspiraciones; perdurar, albergar, posibilitar. Ésas podrían ser las virtudes de una buena casa, aún a riesgo de que la puesta en práctica de estas bondades signifique la lenta desaparición de la estructura original. ¿Cómo el arquitecto puede resistir esa condena? La aceptación de cierta independencia y vitalidad de la arquitectura, que se desencadena apenas terminada la obra, podría contener una respuesta. Palabras clave: Arquitectura - Perí, vivienda social, concursos de arquitectura, autoconstrucción, barrio, crecimiento progresivo.

To adapt to the inhabitant's needs, give space to bis dream to last, shelter, enable: this could describe the qualities of a good house, although putting them into practice may mean the gradual disappearance of the original structure. ¿ How an the architect resist this sentence? Perhaps by accepting a degree of independence and vitality in the architecture, set free the moment the work is finished. d-it-yourself construction, neighborhood, progressive growth

\section{Presentación}

El presente artículo corresponde al material elaborado a partir de la tesis de investigación "Arquitectura, vida y transformaciones" para su exposición en la IV Bienal Iberoamericana de Arquitectura, realizada en Lima en octubre del 2004, cuyo tema giró en torno a la vivienda social en Iberoamérica.

Compuesto por 26 propuestas, y a más de treinta años de su inicio, la experiencia del Proyecto Piloto 1 del PREVI ${ }^{1}$ en Lima, Perú, es materia de investigación por la valiosa complejidad de factores que intervienen: el collage de proyectos, la variedad tipológica, el carácter experimental de la propuesta y el tiempo que lleva sometida a diferentes intervenciones autogestionadas.

Viajamos a Lima a reconstruir el proceso de transformación de los proyectos realizados a partir de levantamientos de croquis sobre los planos originales, levantamientos fotográficos y entrevistas a las familias que los habitan. Desde el levantamiento de las viviendas actuales y el cruce con la historia familiar, se descubre la articulación entre el proyecto del arquitecto y el proyecto del habitante. La investigación enfatiza el proceso de la vivienda, vinculada estrechamente a la historia familiar.

\section{El Proyecto Experimental de Vivienda PREVI}

Durante la década de los sesenta, la precariedad de los asentamientos espontáneos y el deterioro de algunos sectores de Lima estimularon una serie de iniciativas del gobierno peruano y del PNUD 2 destinadas a incorporar a las políticas de vivienda los resultados de tres proyectos piloto. De ellos,
1 Proyecto Experimental de Vivienda, desarrollado entre 1967 y 1978 segu concurso, 1967-1969; desarrollo 19701972; construcción, 1972-1976; entrega de las viviendas, 1978-1979 2 Programa de las Naciones Unidas para el Desarrollo. ${ }^{3}$ Los 13 equipos invitados fueron: Esquerra, Samper, Sáenz, Urdaneta Colombia, Atelier 5/Suiza, Korhonend Finlandia, Correa/lndia, Kikutake, Mak, Kurokaw/Japon, Iniguez de Onzoñ, Vasquez de Castro//España, Hansen, Hatloy/Polonia, Aldo van Eyck/Holand, Christopher Alexander/EE UU,

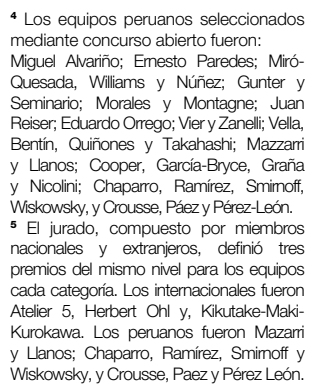
Mediante concurso abierto fueron: Lesada, Willians y Núñez: Gunter y

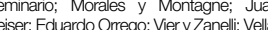

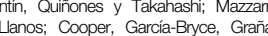
Nicolini; Chaparo, Ramirez, Smimo El jurado, compuesto por miembros remios del mismo nivel para los equipos Aelier 5, Herbert Ohl y, Kikutake-MakLanos; Chaparo, Ramírz, Smimoff y

el de mayor connotación fue el Proyecto Piloto 1, que a través de un concurso internacional ${ }^{3} \mathrm{y}$ otro nacional ${ }^{4}$, materializó las recientes discusiones en torno a la arquitectura de la vivienda -en cierta medida, una contrapropuesta a los paradigmas modernos de la vivienda multifamiliar-. Entre los conceptos propuestos por el concurso estaban la racionalización, modulación, tipificación, crecimiento progresivo, flexibilidad y función. Cada tipología planteaba distintas versiones para diferentes grupos familiares, además de sistemas de crecimiento de las unidades.

$\mathrm{Si}$ bien el objetivo original de ambos concursos ${ }^{5}$ era la construcción de 1.500 viviendas a partir de los proyectos ganadores, el interés demostrado por el jurado ${ }^{6}$ se tradujo en un hecho fortuito pero decisivo para el futuro de PREVI: se construyeron las 26 propuestas recibidas en un conjunto de 500 unidades. El énfasis estuvo en la exploración arquitectónica y técnica propuesta por los proyectos, para lo cual se instaló una planta del ININVI $^{7}$ en el lugar para la prefabricación de los componentes constructivos y la posterior asistencia a los usuarios en la ampliación de sus casas.

La ejecución de PREVI se vio enfrentada a circunstancias políticas que retrasaron diversas fases de su desarrollo; las crisis institucionales de los setenta fueron un obstáculo para la continuidad del proyecto. Aun así, el Grupo de Desarrollo ${ }^{8}$ fue capaz de llevar a cabo los proyectos, y hacer un seguimiento detallado de los procesos constructivos demostrando su viabilidad en términos económicos, entre otros aspectos. Las tensiones PREVI - gobierno de turno tuvieron como resultado final un retraso en el proceso de adjudicación de las viviendas, discontinuidad en los procesos de asesoría y, finalmente, un cierto olvido voluntario de la importancia de la experiencia.

\section{Tiempo, casos}

I. La separación del peatón y el automóvil promueve la consolidación de comunidades vecinales

Las plazas son la unidad de vecindario y de espacio público de PREVI. Su tamaño en relación a un cierto número de viviendas ha facilitado la organización de los vecinos, que se han encargado de mantenerlas, cualificando tanto el barrio como la vivienda.

El Grupo de Desarrollo de PREVI propuso una estructura de pequeñas plazas, interconectadas por pasajes peatonales, que articulan las múltiples formas de agrupación de los proyectos originales. Se fundó de esa manera un orden urbano basado en una unidad social y espacial, las plazas de vecindad. Las plazas y pasajes construyen un interior peatonal
6 El jurado estuvo compuesto por José Antonio Coderch (España), Halldor Gunnlogsson (Dinamarca), Peter Land
(O.N.U.). Emest Weissmann (O.N.U) Carl Koch (EE.UU., UIA), Manuel Valega (Perú), Ricardo Malachowski (Perú), Eduardo Barclay (Perú) y los asesores Darío rerizán El Grupo de Desarrollo estuvo integrado por especialistas internacionales y nacionales, algunos de los cuales fueron ambiando en las etapas del proyecto. En las etapas iniciales, la asesonia

and (arquitecto, asesor principall) y Álvaro Gonzalez (Peru) y A Avaro Ortega (O.N.U). Ortega (arquitecto, consultor interregional ONU) como supenisor. En la directiva (arquitecto), Carlos Morales Macchiavello (arquitecto), Oscar Pacheco (arquitecto) entre los 10 Directores que tuvo PREVI. Raquel Barrionuevo de Machicao (ingeniera civil e ingeniera sanitaria) y Javier en todas las fases del proyecto induvendo la Evaluación Integral PREVI.

con características notables. Su consolidación se debe a múltiples razones; el cuidado de la vegetación a cargo de la comunidad, una condición de silencio y tranquilidad interior, el juego de los niños bajo el cuidado de los vecinos y un zócalo continuo que soporta la variedad tipológica hacen de las plazas un valioso rincón urbano.

\section{Las potencialidades de una vivienda varían} de acuerdo a su localización en el conjunto

El barrio no es un tejido homogéneo que se corta donde termina el predio. La composición del sistema urbano puede definir qué tan buena es la costura que después hará la ciudad con un nuevo barrio. El soporte urbano y la incorporación de usos que superan la escala de proyecto original hacen de PREVI un barrio funcionalmente integrado al resto de la ciudad.

La respuesta a la necesidad de combinar 26 tipologías distintas fue la construcción de un soporte urbano a partir de elementos tan disímiles pero complementarios como son las plazas de vecindad, los pasajes peatonales, los accesos vehiculares, estacionamientos y un parque. Esta composición hizo que cada casa gozara de un rol distinto en el barrio, lo que se tradujo en nuevos programas incorporados a la vivienda relativos a su posición en PREVI. Sobre las calles vehiculares se concentra el comercio, así como sobre la avenida peatonal; alrededor del parque se ubican los nuevos colegios que lo usan de patio.

III. Los grupos familiares son diversos y múltiples, sus procesos determinan el crecimiento de la vivienda

Las políticas públicas de vivienda han tenido una visión homogénea y tipificada del usuario, que ha rigidizado el proyecto de vivienda económica. Éste, multiplicado varias veces en la ciudad, entra en conflicto con la variabilidad de las realidades de la familia, por lo tanto del barrio, por la tanto de la ciudad. El cruce de las historias familiares con la transformación de la vivienda devela una de las claves del proceso: el patrón de evolución familiar es uno de los motores principales para que cada familia vaya satisfaciendo requerimientos que varían con los años de acuerdo al siguiente etapamiento:

Instalación, la familia introduce modificaciones menores para asegurar la propiedad y definir la propia imagen de la casa; Densificación, la familia crece e incorpora nuevos núcleos, lo que demanda el mayor esfuerzo constructivo, se construyen principalmente dormitorios y nuevos baños, además de la incorporación de otros usos; Consolidación y diversificación; además de las últimas inversiones en 

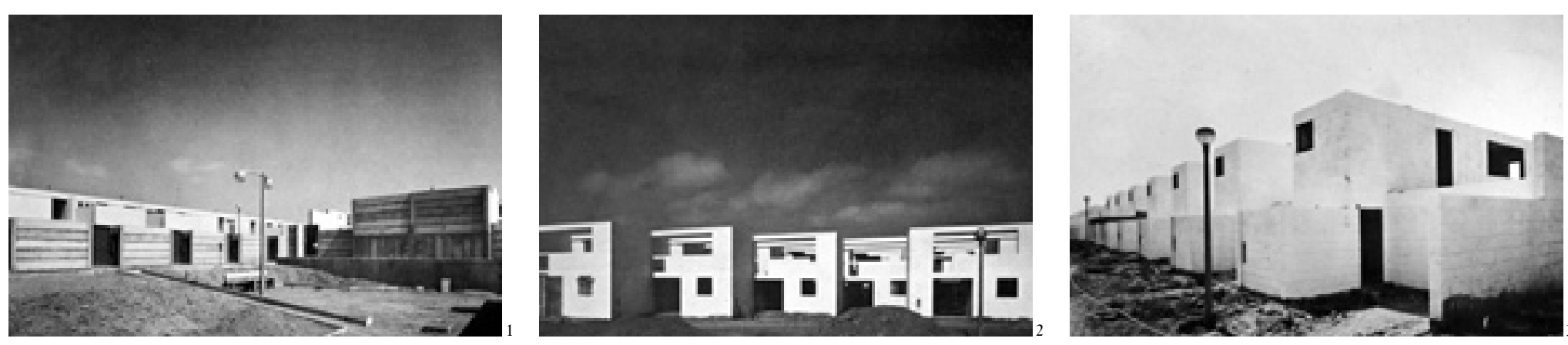

$\begin{array}{lcc}\text { Todas las imágenes } & \text { 1 Plaza de Atelier 5,1978 } & \text { 4 Plaza de Atelier 5, 2003 } \\ \text { pertenecen al archivo de los } & \text { 2 } \text { Proyecto de } & \text { 5 Proyecto de } \\ \text { autores, excepto las fotos } & \text { Charles Correa, 1978 } & \text { Charles Correa, 2003 } \\ \text { de 1978 obtenidas de las } & \text { 3 Esquina Kikutake-Maki- } & \text { 6 Esquina Kikutake-Maki- } \\ \text { publicaciones hechas por el } & \text { Kurokawa, 1978 } & \text { Kurokawa, 2003 } \\ \text { ININVI. } & \end{array}$
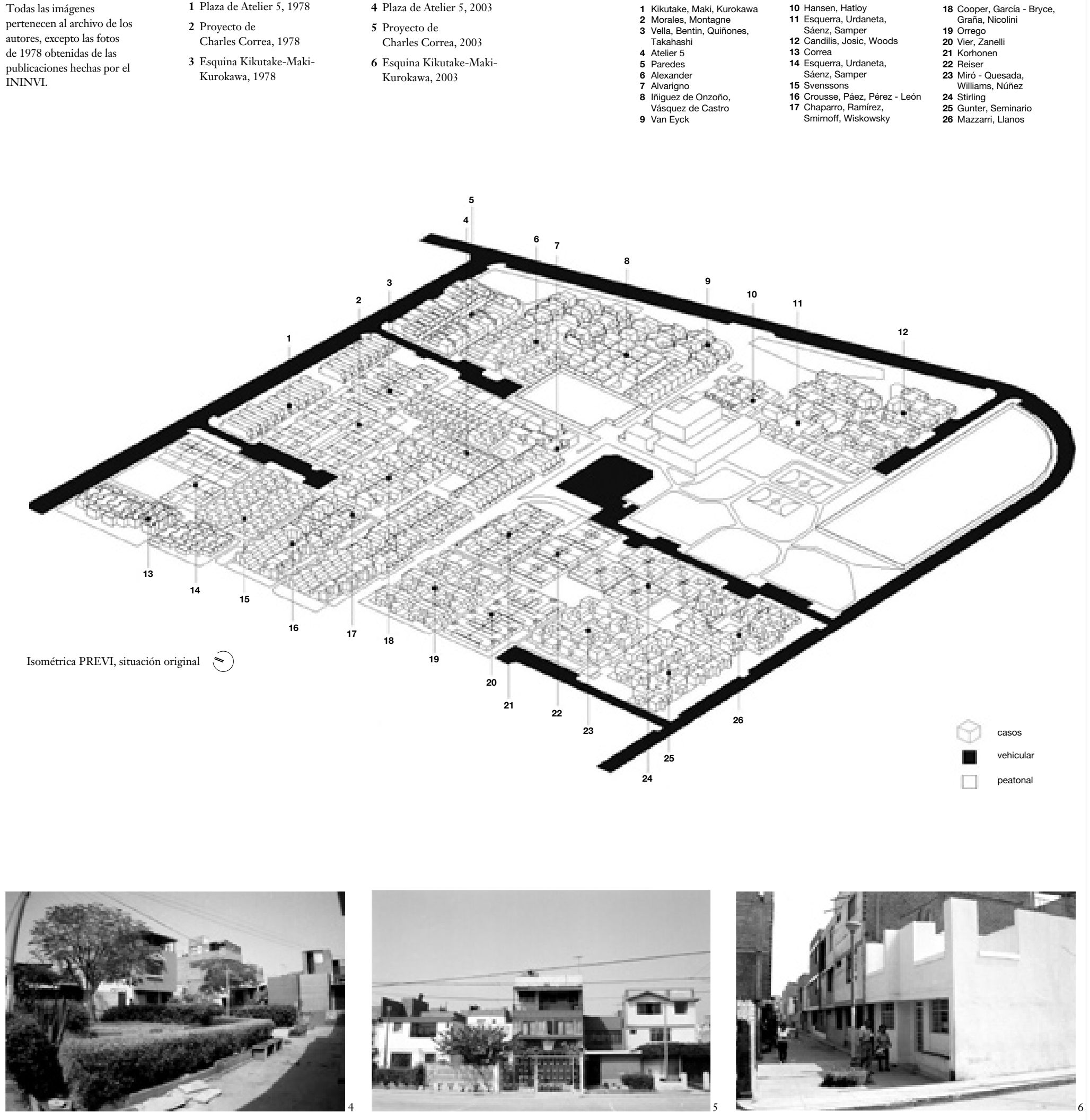


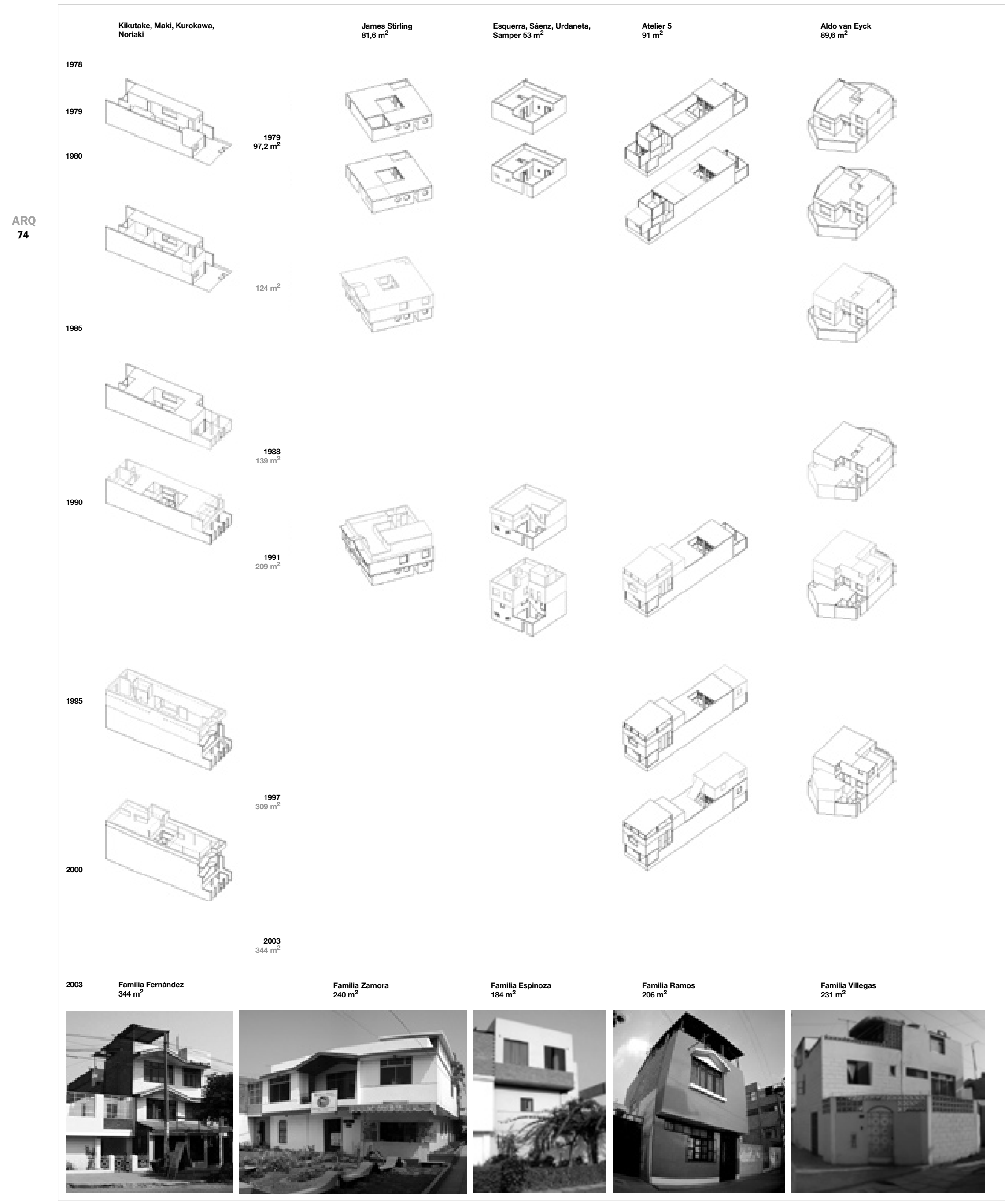

ARQ Ensayos y documentos Essays and documents 
Miró-Quesada,

$\underset{89,6 \mathrm{~m}^{2}}{\text { Wuiliams, Núnez }}$

Morales Montagne

Charles Correa

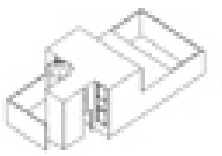
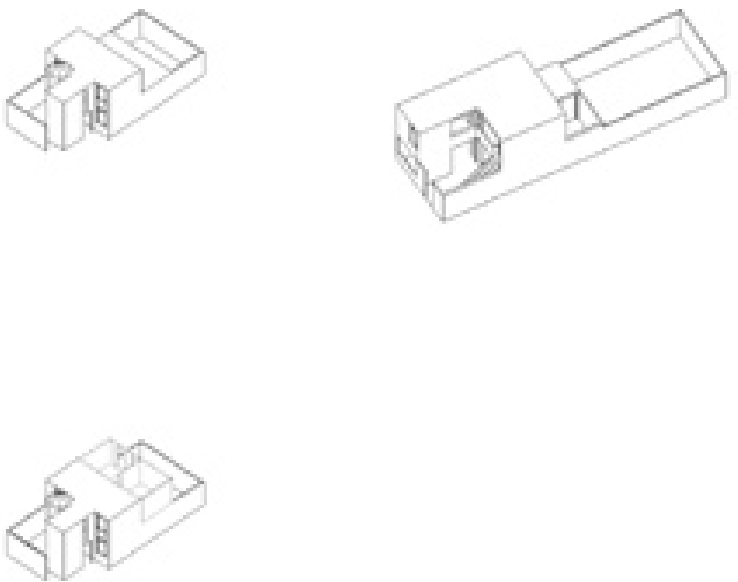

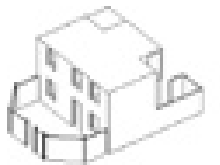

- $\frac{d y}{40}$

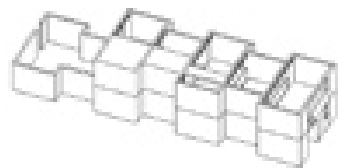

1979

1980

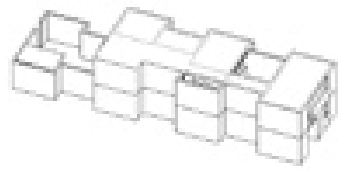

(4)
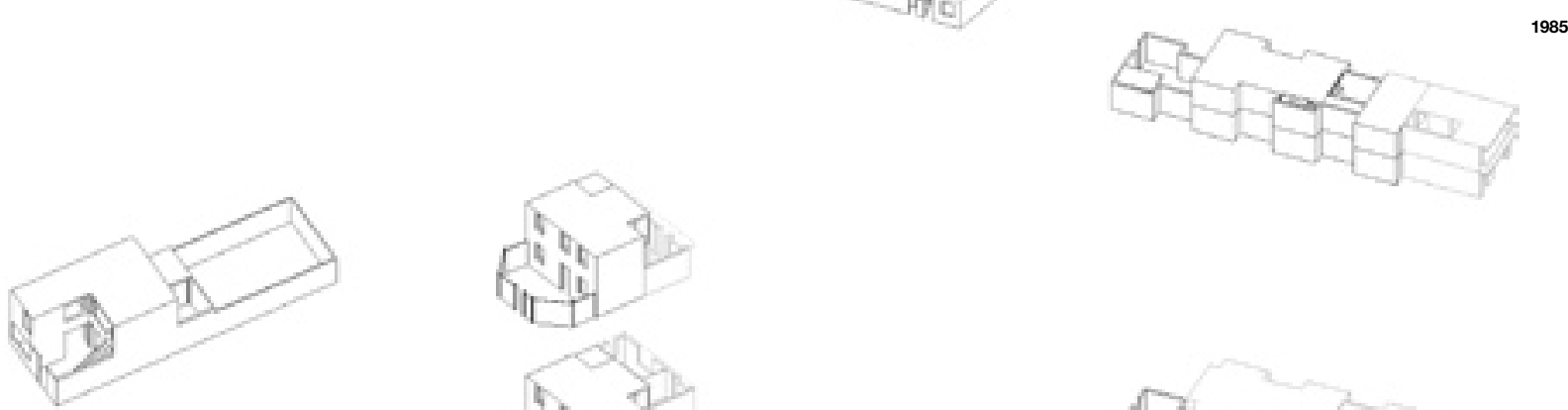

Finit
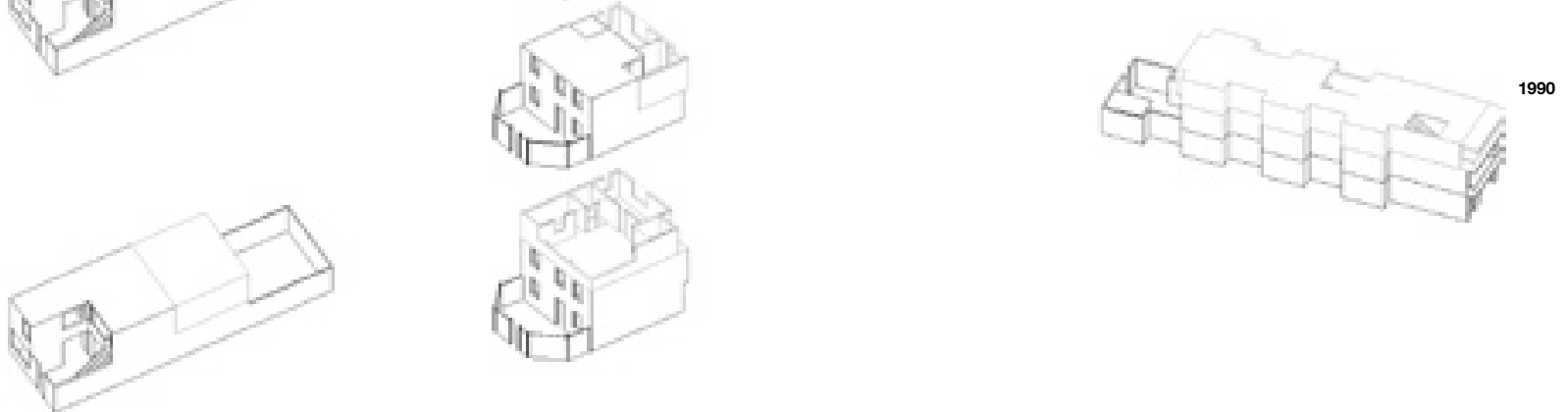

1985
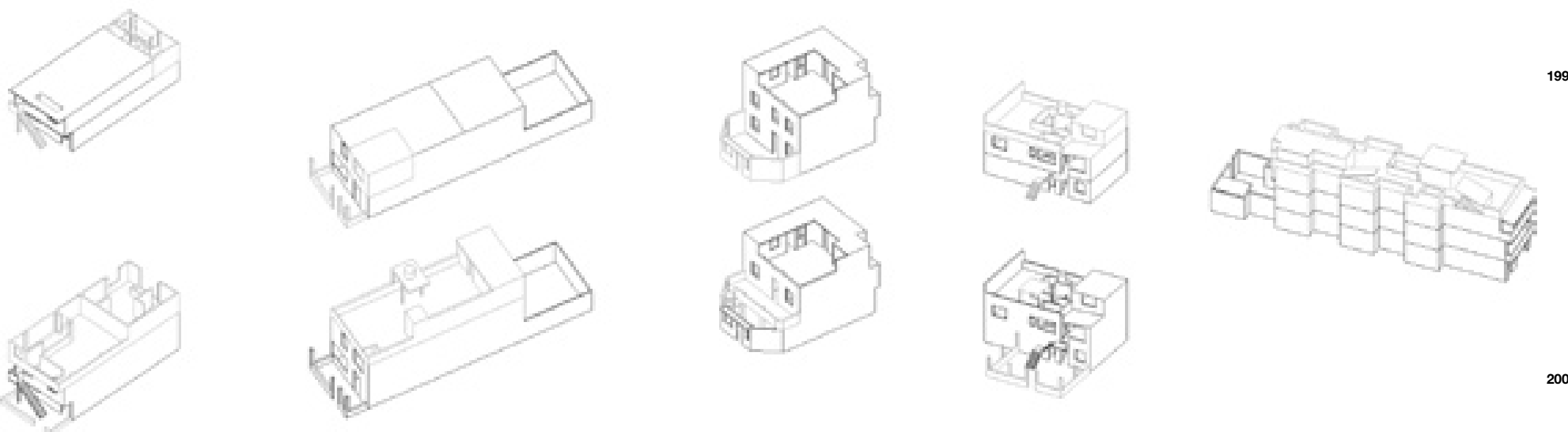

1995

2000
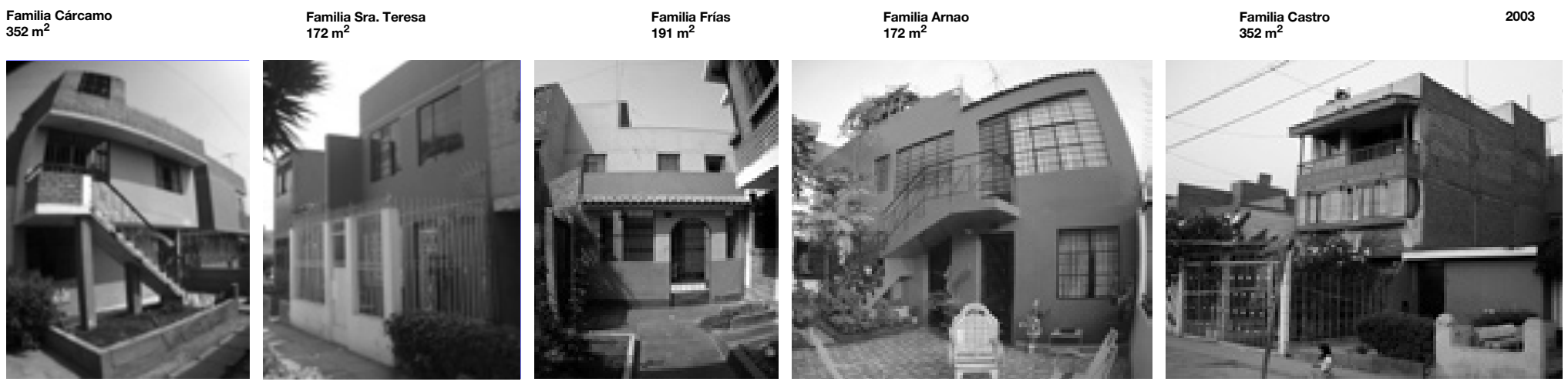

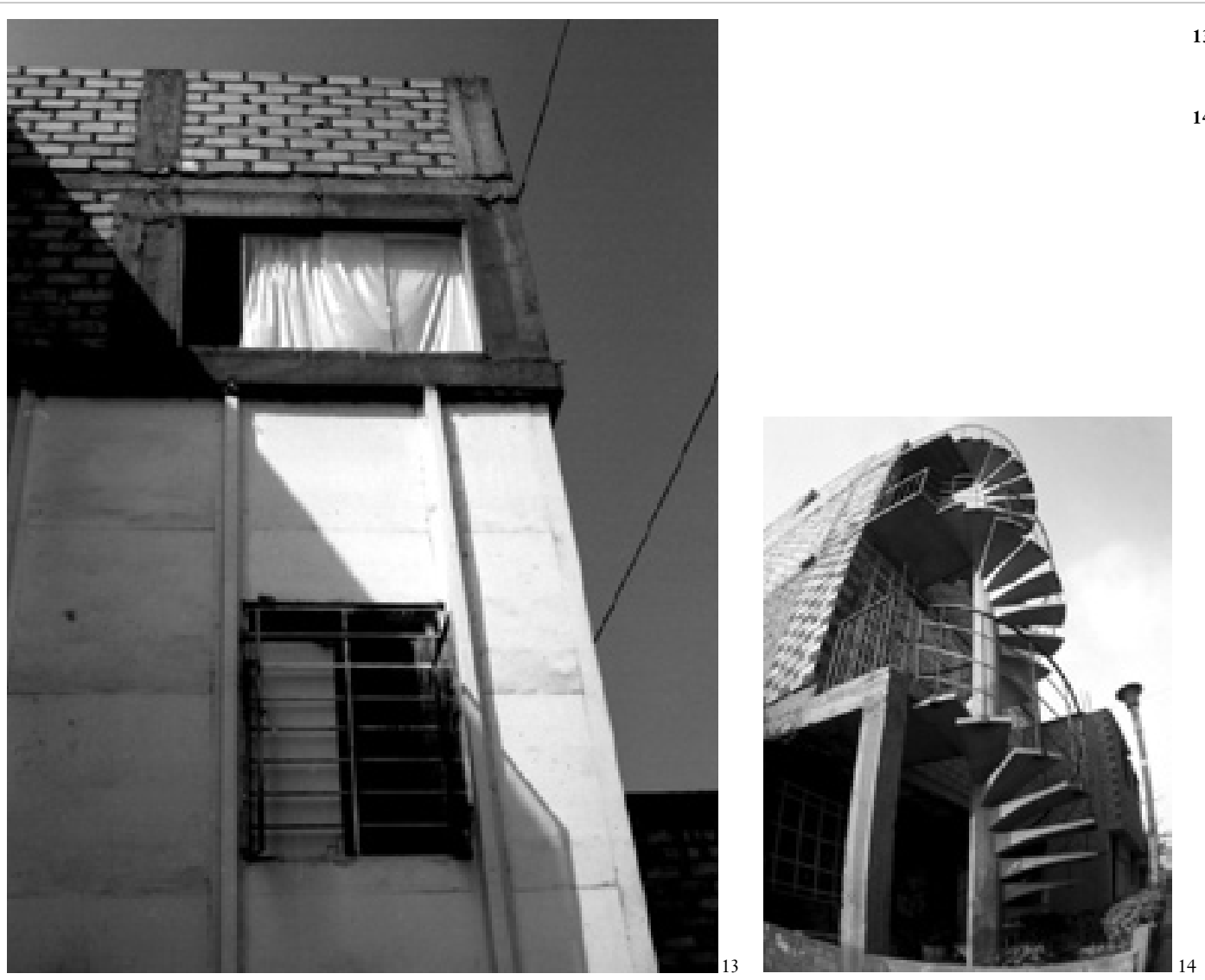

13 Superposición de materiales sistemas constructivos, sobre proyecto de Svenssons

14 Elementos prefabricados; escalera original de Svenssons usada en proyecto de Paredes
- El clima desértico, con casi nulas precipitaciones y la inestabilidad propia
de la costa, hacen de Lima una ciudad de la costa, hacen de Lima una ciudad
donde es posible el estado de construcción permanente. Los techos son habitables hasta que se transforman en recintos que agregan una nueva azotea. terminaciones, la casa se subdivide funcionalmente en departamentos para varias familias.

La comprensión de este patrón demanda pensar más que en un usuario tipo en un grupo familiar diverso y dinámico en el tiempo. El patrón Instalación / Densificación / Diversificación arroja resultados como el de la vivienda multifamiliar, realidad que no es recogida por las políticas públicas en términos del habitante.

\section{La vivienda inicial es un soporte para una} nueva imagen y nuevos usos

La casa en PREVI es una plataforma de transformaciones, la construcción de un zócalo sobre el cual se superpone naturalmente la ciudad. Las virtudes de este proceso están relacionadas con el potencial de la vivienda de ser un artefacto de renta: las transformaciones llevadas a cabo por el usuario son inversiones que pueden reportar ingresos a la familia, a través de un negocio o el alquiler de parte de la casa.

Por otro lado, la plataforma significa la posibilidad de que cada familia construya la propia imagen, incorporando con la simple mímesis la casa al paisaje completo de la ciudad. De esta manera, uno que podía ser identificado como un barrio social-con su connotación institucional- es ahora parte de un barrio popular integrado.

Las consecuencias de este concepto sobre el proyecto se pueden recoger en las permanencias y la relación bolgura/rigidez. Permanencias, son aquellos elementos constructivos o programáticos que perduran en el proceso de transformación. El esquema holgura/rigidez establece la disposición de los elementos estructurales y los espacios del cual dispone el habitante para incorporar nuevas estructuras. Estas son las reglas que el habitante debe interpretar para poder ampliar la casa de acuerdo a ciertos parámetros del proyecto; de ahí la importancia de la disposición de estos elementos en la plataforma inicial, ya que de ellos dependen las posibilidades de la casa final.

V. El crecimiento en torno a un patio asegura las condiciones medioambientales de la vivienda El patio juega un rol principal en las casas, no sólo en términos espaciales sino también en la claridad que establece para el proceso de crecimiento. Las condiciones de adosamiento de las viviendas hacen que en muchos casos sea el patrimonio principal de la casa, siendo una de las permanencias más importantes. El habitante es capaz de interpretar el rol que cumple y es en definitiva el elemento que asegura un crecimiento sin poner en juego las cualidades ambientales originales de la casa9.

\section{PREVI, barrio y ciudad}

\section{Ciudad collage}

La ciudad entendida como collage -no sólo compuesta de diferentes intervenciones macro, sino también de un sinnúmero de microtransformaciones- aporta a la complejización del tejido social y la integración urbana de los barrios populares. Entonces, la ciudad collage es una ciudad viva, una ciudad compleja.

Barrio conjunto de casas

Es preciso entender un barrio no como un conjunto de casas, sino como una asociación de equipamientos y casas, donde la estructura urbana y la arquitectura son vitales para detonar una exitosa evolución del barrio y las viviendas. El colegio de PREVI potencia el uso de los espacios públicos con el desarrollo de actividades propias en el parque, generando una superposición de actividades que le dan una gran intensidad urbana al barrio.

Vecinal $=$ unidad urbana

La estrecha relación entre unidad urbana -la plaza- y unidad social -la comunidad vecinal capaz de organizarse- promueve la apropiación y cuidado del espacio público colectivo.

En contraste con la homogeneidad de las urbanizaciones institucionales convencionales, el sistema de plazas y pasajes peatonales representa la permanencia de un trazado complejo y rico en situaciones urbanas diversas.

"Los 35 años de PREVI merecen ser celebrados como lección de arquitectura: de humildad, porque los derroteros del proyecto jamás quedarán congelados a la visión original del autor en todos sus aspectos; y de confirmación, por cuanto las obras iniciales, estrategias, postulados y la convicción de sus autores y gestores dieron inicio a la formación de un barrio urbano desde muchos puntos de vista destacable". Rodrigo Pérez de Arce, 2004. ARQ

Agradecimientos a Rodrigo Pérez de Arce, Fernando Pérez Oyarzun, Raquel Barrionuevo de Machicao y Wiley Ludeña, por la guía y colaboración en la pesquisa. A las familias de PREVI, por permitirnos visitar sus casas.

Bibliografía: Entrevista de los autores con Wiley Ludeña, lunes 28 de abril de 2003, Lima. / Entrevista de los autores con Raquel Barrionuevo de Machicao, viernes 9 de mayo de 2003, Lima. / VV.AA.; Publicación PREVI, vol. 1 - 24. Ministerio de Vivienda del Perú e Instituto de Investigación y Normalización de la Vivienda ININVI, Lima, 1979. / VV.AA.; PREVI 20 Años, Resultados y Conclusiones. Ministerio de Vivienda del Perú e Instituto de Investigación y Normalización de la Vivienda ININVI, Lima, s.d. 